\title{
Prolonged extracorporeal membrane oxygenation-assisted support provides improved survival in hypothermic patients with cardiocirculatory arrest
}

Elfriede Ruttmann, MD, ${ }^{a}$ Annemarie Weissenbacher, MD, ${ }^{a}$ Hanno Ulmer, PhD, ${ }^{b}$ Ludwig Müller, MD, ${ }^{a}$ Daniel Höfer, MD, ${ }^{a}$ Juliane Kilo, MD, ${ }^{a}$ Walter Rabl, MD, ${ }^{c}$ Birgit Schwarz, MD, ${ }^{d}$ Günther Laufer, MD, ${ }^{a}$ Herwig Antretter, MD, ${ }^{a}$ and Peter Mair, MD ${ }^{\mathrm{d}}$

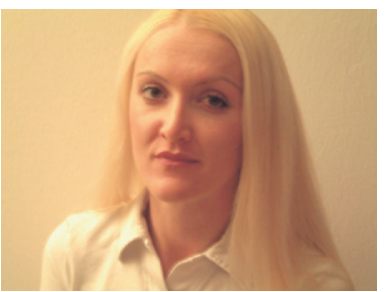

Dr Ruttmann

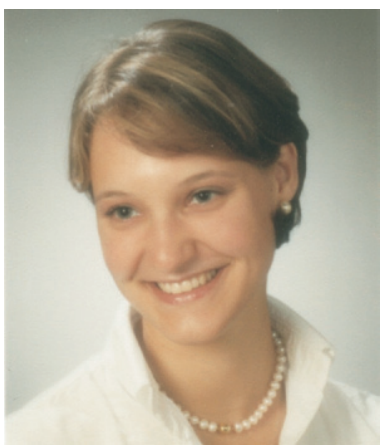

Dr Weissenbacher

From the Departments of Cardiac Surgery, ${ }^{\mathrm{a}}$ Medical Statistics, Informatics and Health Economy, ${ }^{\mathrm{b}}$ Forensic Medicine, ${ }^{\mathrm{c}}$ and Anesthesiology and Intensive Care Medicine, ${ }^{\mathrm{d}}$ Innsbruck Medical University, Tyrol, Austria.

Presented at the World Congress of Cardiology, September 2006, Barcelona, Spain.

Received for publication Jan 14, 2007; revisions received March 12, 2007; accepted for publication March 22, 2007.

Address for reprints: Elfriede Ruttmann, MD, Department of Cardiac Surgery, Innsbruck Medical University, Anichstrasse 35, 6020 Innsbruck, Austria (E-mail: elfriede. ruttmann@i-med.ac.at).

J Thorac Cardiovasc Surg 2007;134:594-600 $0022-5223 / \$ 32.00$

Copyright (C) 2007 by The American Association for Thoracic Surgery

doi:10.1016/j.jtcvs.2007.03.049
Objective: Extracorporeal circulation is considered the gold standard in the treatment of hypothermic cardiocirculatory arrest; however, few centers use extracorporeal membrane oxygenation instead of standard extracorporeal circulation for this indication. The aim of this study was to evaluate whether extracorporeal membrane oxygenation-assisted resuscitation improves survival in patients with hypothermic cardiac arrest.

Methods: A consecutive series of 59 patients with accidental hypothermia in cardiocirculatory arrest between 1987 and 2006 were included. Thirty-four patients $(57.6 \%)$ were resuscitated by standard extracorporeal circulation, and 25 patients $(42.4 \%)$ were resuscitated by extracorporeal membrane oxygenation. Accidental hypothermia was caused by avalanche in 22 patients (37.3\%), drowning in 22 patients $(37.3 \%)$, exposure to cold in 8 patients $(13.5 \%)$, and falling into a crevasse in 7 patients (11.9\%). Multivariate logistic regression analysis was used to compare extracorporeal membrane oxygenation with extracorporeal circulation resuscitation, with adjustment for relevant parameters.

Results: Restoration of spontaneous circulation was achieved in 32 patients (54.2\%). A total of 12 patients $(20.3 \%)$ survived hypothermia. In the extracorporeal circulation group, $64 \%$ of the nonsurviving patients who underwent restoration of spontaneous circulation died of severe pulmonary edema, but none died in the extracorporeal membrane oxygenation group. In multivariate analysis, extracorporeal membrane oxygenation-assisted resuscitation showed a 6.6-fold higher chance for survival (relative risk: $6.6,95 \%$ confidence interval: $1.2-49.3, P=.042$ ). Asphyxia-related hypothermia (avalanche or drowning) was the most predictive adverse factor for survival (relative risk: $0.09,95 \%$ confidence interval: $0.01-0.60, P=.013$ ). Potassium and $\mathrm{pH}$ failed to show statistical significance in the multivariate analysis.

Conclusions: Extracorporeal rewarming with an extracorporeal membrane oxygenation system allows prolonged cardiorespiratory support after initial resuscitation. Our data indicate that prolonged extracorporeal membrane oxygenation support reduces the risk of intractable cardiorespiratory failure commonly observed after rewarming.

B ecause of the protective effects of deep hypothermia, prolonged resuscitation in patients with severe accidental hypothermia is recommended. ${ }^{1}$ Support with extracorporeal circulation (ECC) is considered the gold standard in the treatment of hypothermic patients with cardiocirculatory arrest; however, survival of these patients is still poor., ${ }^{2,3}$ Several years ago, portable extracorporeal membrane oxygenation (ECMO) became commercially available and was introduced as an alternative treatment option to ECC for emergency mechanical circulatory support in cardiothoracic surgery. 


$$
\begin{aligned}
& \text { Abbreviations and Acronyms } \\
& \begin{aligned}
\mathrm{CI} & =\text { confidence interval } \\
\mathrm{ECC} & =\text { extracorporeal circulation } \\
\mathrm{ECMO} & =\text { extracorporeal membrane oxygenation } \\
\mathrm{ROSC} & =\text { return of spontaneous circulation } \\
\mathrm{RR} & =\text { relative risk }
\end{aligned}
\end{aligned}
$$

The successful use of ECMO has been reported in normothermic patients undergoing noncardiac surgery who have cardiogenic shock $\mathrm{k}^{4,5}$ and in patients undergoing open surgery who cannot be weaned from cardiopulmonary bypass. ${ }^{6,7}$

ECMO has several advantages compared with standard ECC equipment: (1) Cannulation can be performed percutaneously (even outside of the operating room during external chest compression) and can be used for single pulmonary support when used as a venovenous system. ${ }^{8}$ (2) ECMO requires lower levels of anticoagulation and can be used without systemic anticoagulation in case of major bleeding. (3) Prolonged extracorporeal support for hours and days is possible. ${ }^{9}$ (4) The system can be transferred within hospitals. (5) Negative pressure on the venous cannulas allows higher extracorporeal flow rates.

In contrast with ECC, ECMO support cannot decompress a severely impaired left ventricle because it is a closed system.

In hypothermic patients with cardiocirculatory arrest, ECC is used during cardiopulmonary resuscitation in several centers. ${ }^{10-13}$ The limited support time of ECC, because of the inflammatory activation and hemolysis, might not be sufficient to adequately treat reperfusion injury. ${ }^{14}$ Reperfusion edema is a well-known phenomenon in lung transplantation, frequently requiring prolonged ECMO support to provide sufficient oxygenation. ${ }^{15,16}$

Although previous case series of hypothermia have reported successful rewarming with the use of ECC, there has never been a study published investigating the effect of ECMO-assisted rewarming in hypothermic patients with cardiocirculatory arrest. Most previous studies could not evaluate such an effect because of the limited sample size or unavailability of ECMO support. ${ }^{17,18}$ During the past few years, we have observed a marked improvement in survival in hypothermic patients resuscitated by ECMO support that could not be solely explained by the more restrictive use of extracorporeal rewarming in patients with concomitant asphyxia (eg, in avalanche accidents). Considering these advantages, we hypothesized that prolonged ECMO support is superior in the treatment of accidental hypothermia with cardiocirculatory arrest.

\section{Materials and Methods}

We retrospectively analyzed a consecutive series of 59 patients with accidental hypothermia and cardiocirculatory arrest who were admitted to the Medical University Innsbruck between 1987 and 2006. Inclusion criterion for this study was attempted rewarming with the extracorporeal-assisted technique in patients with hypothermic cardiac arrest. Permission for data analysis and data protection of the presented patients was given by the local institutional review board.

Thirty-four patients $(57.6 \%)$ were resuscitated by ECC (ECC group, 1987-1996), and 25 patients (42.4\%) were resuscitated by venoarterial ECMO (ECMO group, 1996-2006).

At admission, 31 patients $(52.5 \%)$ had asystole, 25 patients (42.4\%) had ventricular fibrillation, and 3 patients $(5.1 \%)$ had pulseless electrical activity. Hypothermia was caused by avalanche in 22 patients (37.3\%), drowning in 22 patients $(37.3 \%)$, exposure to cold in 8 patients $(13.5 \%)$, and falling into a crevasse in 7 patients $(11.9 \%)$.

\section{Patient Management and Cannulation Technique}

Before patients were admitted to the emergency department, anesthesiologists, cardiac surgeons, and perfusionists were frequently instructed to organize a rescue team for extracorporeal rewarming.

In the ECMO group, patients with return of spontaneous circulation (ROSC) remained on the extracorporeal assist device up to several days until cardiopulmonary function was stabilized (Figure 1).

Patients who received ECC were cannulated femorally by an open surgical technique using purse-string sutures for vessel preparation. In smaller children $(n=6,17.6 \%)$, central ECC cannulation by sternotomy was performed. In the ECMO group, femoral cannulation was attempted percutaneously and was successful in 14 patients $(66.7 \%)$. Routinely, an additional $5 \mathrm{~F}$ cannula was distally inserted into the femoral artery for leg perfusion in all patients receiving ECMO. Two small children underwent ECMO cannulation with a median sternotomy. If repeated efforts of percutaneous insertion failed, groin incision and direct cannulation with purse-string sutures were performed.

After initiation of cardiopulmonary bypass, the patient was kept at the admission temperature for the first 5 minutes, and then active rewarming was started with a warming rate of approximately $7^{\circ} \mathrm{C}$ per hour. Body core temperature was measured by tympanum temperature during the rewarming period. The active rewarming period took approximately 1.5 hours until normothermia was reached; the maximum gradient between core temperature and water bath of the heat exchanger was kept at less than $8^{\circ} \mathrm{C}$. At a core temperature of approximately $27^{\circ} \mathrm{C}$, active (and if necessary recurrent) defibrillation and buffering with sodium bicarbonate were started.

The ECC system consisted of a roller pump (Stöckert, Bonn, Germany) and an oxygenator with an integrated heat exchanger (from 1987 to 1992, a bubble oxygenator, Polystan AS, Denmark; from 1993 to the present, a membrane oxygenator, Jostra AG, Verloese, Denmark). Polyvinyl cannulas (Stöckert, Bonn, Germany) were used for peripheral femoral cannulation.

The ECMO system consisted of a centrifugal pump (Biopump, Bio-Medicus, Eden, Prairie, Minn), a hollow-fiber oxygenator 


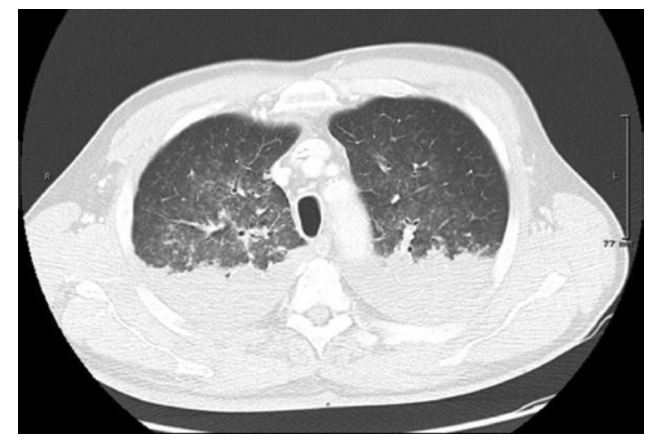

Figure 1. Severe pulmonary edema in a person rescued from a crevasse after successful ECMO-assisted resuscitation. Prolonged ECMO assistance was necessary for 27 hours because of massive reperfusion edema of the lung. Computed tomographic scan was performed after the patient was normothermic. ECMO, extracorporeal membrane oxygenation.

(Maxima, Medtronic Bloodsystem, Anaheim, Calif), polyvinyl chloride tubing, percutaneous arterial and venous cannulas (Medtronic Inc, Minneapolis, Minn), and an integrated heat exchanger (Stöckert, Bonn, Germany).

\section{Statistical Analysis}

Possible associations between successful resuscitation and potentially predictive clinical features were assessed by means of univariate and multivariate analyses. To test for univariate differences in categoric variables, the Pearson chi-square test or Fisher exact test (when appropriate) was applied. Continuous variables were tested with the Student $t$ test or Mann-Whitney $U$ test (if assumption of a Gaussian distribution was not fulfilled). Multivariate logistic regression analysis was performed to determine the relative risk (RR) and 95\% confidence intervals (CIs) for potential predictors of surviving extracorporeal resuscitation. Thereby, the selection of variables was based on univariate comparisons (entry criteria $P<.05)$ and clinical relevance.

\section{Results}

The mean age of patients was $31 \pm 18$ years, mean body core temperature at admission was $24.5^{\circ} \mathrm{C} \pm 3.7^{\circ} \mathrm{C}$, and mean burial/submersion time was $93.8 \pm 104.6$ minutes. At admission, the mean lactate level was $129.8 \pm 60.9 \mathrm{mg} / \mathrm{dL}$, mean potassium level was $7.4 \pm 4.1 \mathrm{mmol} / \mathrm{L}$, and mean $\mathrm{pH}$ was $6.68 \pm 0.27$. During the rewarming period, reestablishment of sinus rhythm during extracorporeal rewarming was achieved in 33 patients $(56.0 \%)$. Weaning from the assist device (ECC or ECMO) after ROSC was possible in 32 patients (54.2\%). A total of 12 patients (20.3\%) survived hypothermia and were discharged from the hospital. The majority of patients with ROSC died within 2 days. Of the surviving patients $(\mathrm{n}=12)$, the median intensive care unit stay was 15 days (range 5 days to 6 weeks) and the median hospital stay was 29 days (18-65 days). Initial hemofiltra- tion was necessary in 5 patients; the remaining survivors redeveloped sufficient kidney function immediately after reaching normothermia. Tracheostomy was required in 2 patients after prolonged invasive ventilation to successfully wean the patient from the respirator. There were no neurologic complications during the hospital stay. Eleven patients regained full neurophysiologic and intellectual integration into their normal lives. One surviving child (6 years old, survivor of drowning accident) was attending a special school because of a single intellectual impairment without any apparent neurologic dysfunction.

The characteristics of the patients according to the applied extracorporeal technique are summarized in Table 1.

\section{Evaluation of Predictors for Successful Resuscitation}

An overview of the clinical parameters analyzed for their influence on survival is displayed in Table 2.

In the first step, possible clinically relevant factors were analyzed for their influence on survival in the univariate analysis.

The ages of nonsurvivors were not significantly different from those of survivors $(29.5 \pm 16.7$ years vs $38.3 \pm 20.1$ years, $P=.20)$. In addition, male gender was not associated with successful resuscitation $(87.2 \%$ vs $75.0 \%, P=.37$ ). Asphyxia-related cause of hypothermia, either avalanche or drowning as previously described by Walpoth and colleagues, ${ }^{13}$ was significantly associated with death from hypothermia $(85.1 \%$ vs $33.3 \%, P=.001)$. The heart rhythm at admission (asystole vs ventricular fibrillation/pulseless electrical activity) did not have a significant influence on survival (57.4\% vs $33.3 \%, P=.20)$. In comparison with ECC, ECMO-assisted rewarming was significantly associated with improved survival $(34.0 \%$ vs $75.0 \%, P=.02)$. At admission, nonsurvivors had significantly higher potassium levels $(8.1 \pm 4.3 \mathrm{mmol} / \mathrm{L}$ vs $4.9 \pm 1.9 \mathrm{mmol} / \mathrm{L}, P=.014)$ and lower $\mathrm{pH}(6.64 \pm 0.24$ vs $6.80 \pm 0.32, P=.026)$.

The mean burial/submersion time in persons in avalanche and near-drowning accidents was not significantly different with regard to survival (94.7 \pm 86 minutes vs $92.2 \pm 134.6$ minutes, $P=.44)$. In addition, there was no statistical difference in core body temperature $\left(24.5^{\circ} \mathrm{C} \pm\right.$ $0.68^{\circ} \mathrm{C}$ vs $\left.24.2^{\circ} \mathrm{C} \pm 0.35^{\circ} \mathrm{C}, P=.77\right)$, mean lactate levels $(136.9 \pm 64.2 \mathrm{mg} / \mathrm{dL}$ vs $118.1 \pm 59.0 \mathrm{mg} / \mathrm{dL}, P=.57)$, or spontaneous activated clotting time $(201.7 \pm 45.5$ seconds vs $204.7 \pm 32.0$ seconds, $P=.92$ ).

The median ECMO support time was 42 hours (range 18-216 hours) in nonsurvivors and 27 hours in survivors (range 2.5-48 hours).

In the ECC group, the median time from extracorporeal weaning to death was 23 hours (range 0.5-110 hours) in nonsurvivors.

Causes of death in nonsurvivors with a reestablished sinus rhythm are summarized in Table 3. 
TABLE 1. Overview of hypothermic patients who have been resuscitated by the use of extracorporeal techniques ( $\mathrm{n}=59$ patients)

\begin{tabular}{|c|c|c|c|}
\hline & $\begin{array}{l}\text { ECC group (1987-1996) } \\
n=34 \text { patients }\end{array}$ & $\begin{array}{l}\text { ECMO group (1996-2006) } \\
n=25 \text { patients }\end{array}$ & $P$ value \\
\hline Age in years (mean, SD) & $28 \pm 16$ & $36 \pm 19$ & .068 \\
\hline Male gender $(n, \%)$ & $28(82.4 \%)$ & $21(84.0 \%)$ & .72 \\
\hline \multicolumn{4}{|l|}{ Cause of hypothermia-related cardiocirculatory arrest } \\
\hline Avalanche & $16(47.1 \%)$ & $6(24.0 \%)$ & \\
\hline Drowning & $11(32.4 \%)$ & $11(44.0 \%)$ & \\
\hline Crevasse & $3(8.8 \%)$ & $4(16.0 \%)$ & \\
\hline Exposure to cold & $4(11.8 \%)$ & $4(16.0 \%)$ & .34 \\
\hline Body core temperature at admission in degrees Celsius (mean, SD) & $24.6 \pm 2.9$ & $24.2 \pm 4.7$ & .66 \\
\hline Burial/Submersion Time in Minutes (mean, SD) & $94 \pm 85 \min$ & $92 \pm 134 \mathrm{~min}$ & .94 \\
\hline Serum potassium at admission in $\mathrm{mmol} / \mathrm{L}$ (mean, SD) & $7.8 \pm 4.4$ & $6.9 \pm 3.7$ & .45 \\
\hline $\mathrm{pH}$ at admission (mean, SD) & $6.7 \pm 0.27$ & $6.7 \pm 0.28$ & .64 \\
\hline \multicolumn{4}{|l|}{ Cardiac rhythm at admission } \\
\hline Asystole & $18(52.9 \%)$ & $13(52.0 \%)$ & \\
\hline Ventricular fibrillation & $15(44.1 \%)$ & $10(40.0 \%)$ & \\
\hline Pulseless electrical activity & $1(2.9 \%)$ & $2(8.0 \%)$ & .68 \\
\hline
\end{tabular}

ECC, Extracorporeal circulation; ECMO, extracorporeal membrane oxygenation; $S D$, standard deviation.

\section{Evaluation of Independent Predictors for Survival}

In the second step, parameters that showed an influence on survival in the univariate analysis were included in a multivariate logistic regression analysis adjusting for these variables.

The results of the multivariate analysis are summarized in Table 4.

Possible asphyxia-associated cause of cardiocirculatory arrest was the most predictive adverse factor for survival (RR: 0.09, 95\% CI: 0.01-0.60, $P=.013$ ). Extracorporealassisted resuscitation with ECMO was an independent predictor for improved survival (RR: 6.6, 95\% CI: 1.2-49.3,
$P=.042$ ). Potassium and $\mathrm{pH}$ at admission failed to show statistical significance in the multivariate analysis.

According to Locher and colleagues, ${ }^{14}$ who described avalanche victims due to the inferior prognosis of concomitant asphyxia separately, we performed a second subanalysis excluding all avalanche victims $(n=22)$. Furthermore, we wanted to prove the stability of the parameters adjusted in the multivariate regression analysis. In this subanalysis, ECMO-assisted resuscitation was again confirmed to be superior compared with ECC-assisted rewarming regarding survival (RR: 7.6, 95\% CI: 1.14-50.8, $P=.036$ ). Again, serum potassium (RR: $0.75,95 \% \mathrm{CI}: 0.49-1.13, P=.16$ )

TABLE 2. Univariate comparison of possible predictive parameters associated with survival of severe hypothermia associated with cardiocirculatory arrest

\begin{tabular}{|c|c|c|c|}
\hline & $\begin{array}{c}\text { Nonsurvivors } \\
\mathrm{n}=47 \text { patients }\end{array}$ & $\begin{array}{c}\text { Survivors } \\
\mathrm{n}=12 \text { patients }\end{array}$ & $P$ value \\
\hline Age in years (mean, SD) & $29.5 \pm 16.7$ & $38.3 \pm 20.1$ & .20 \\
\hline Male gender $(n, \%)$ & $41(87.2 \%)$ & $9(75.0 \%)$ & .37 \\
\hline Asphyxia-associated cause (Avalanche, Drowning) (n, \%) & $40(85.1 \%)$ & $4(33.3 \%)$ & .001 \\
\hline ECMO-assisted resuscitation (n, \%) & $16(34.0 \%)$ & $9(75.0 \%)$ & .02 \\
\hline Burial/submersion time in minutes (if avalanche or drowning) & $94.7 \pm 86$ & $92.2 \pm 134.6$ & .44 \\
\hline Body core temperature at admission (mean, SD) & $24.5 \pm 0.68$ & $24.2 \pm 0.35$ & .77 \\
\hline Asystole at admission (n, \%) & $27(57.4 \%)$ & $4(33.3 \%)$ & .20 \\
\hline Lactate at admission (mean, SD) & $136.9 \pm 64.2$ & $118.1 \pm 59.0$ & .57 \\
\hline pH at admission (mean, SD) & $6.64 \pm 0.24$ & $6.80 \pm 0.32$ & .026 \\
\hline Potassium at admission (mean, SD) & $8.1 \pm 4.30$ & $4.9 \pm 1.90$ & .014 \\
\hline Activated clotting time in seconds (mean, SD) & $201.7 \pm 45.5$ & $204.7 \pm 32.0$ & .92 \\
\hline
\end{tabular}

$S D$, Standard deviation; ECMO, extracorporeal membrane oxygenation. 
TABLE 3. Causes of death in patients with restoration of spontaneous circulation (ROSC) ( $n=21$ patients)

\begin{tabular}{lcc}
\hline & $\begin{array}{c}\text { ECC group } \\
\mathbf{n}=\mathbf{1 4} \text { patients }\end{array}$ & $\begin{array}{c}\text { ECM0 group } \\
\mathbf{n}=\mathbf{7} \text { patients }\end{array}$ \\
\hline Pulmonary edema & $9(64.3 \%)$ & $0(0 \%)$ \\
Multiorgan failure & $2(14.3 \%)$ & $3(50 \%)$ \\
Brain death & $2(14.3 \%)$ & $3(42.9 \%)$ \\
$\begin{array}{l}\text { Aortic dissection/retroperitoneal } \\
\text { hematoma }\end{array}$ & $0(0 \%)$ & $1(16.7 \%)$ \\
$\begin{array}{l}\text { Bleeding } \\
\text { Median time from ROSC to } \\
\text { death (hours, range) }\end{array}$ & $1(7.1 \%)$ & $0(16.7 \%)$ \\
\hline
\end{tabular}

ECC, Extracorporeal circulation; ECMO, extracorporeal membrane oxygenation; ROSC, return of spontaneous circulation.

and $\mathrm{pH}$ (RR: $2.53,95 \% \mathrm{CI}: 0.055-115.8, P=.63$ ) did not indicate statistical significance.

\section{Discussion}

The results of our study suggest that ECMO-assisted resuscitation in hypothermic cardiocirculatory arrest provides improved survival in patients undergoing extracorporealassisted resuscitation. Although ECC support has to be terminated within several hours because of the massive hemolysis and inflammatory response, ECMO assistance is possible up to several days until the hemodynamic and pulmonary functions of the patient have been stabilized. ${ }^{9,19}$

Furthermore, because both treatment groups (ECC and ECMO) showed a similar restoration of spontaneous circulation but significantly different survivals, our data suggest that the improved outcome of patients who received ECMO was attributable to prolonged cardiopulmonary support. In fact, 9 of 14 patients with ROSC in the ECC group (64\%) died of intractable pulmonary edema within a few hours after rewarming, but none in the ECMO group died (however, severe pulmonary edema was frequently observed during the rewarming period in ECMO-assisted patients too) (Figure 1). We therefore conclude that the improved outcome of ECMO-assisted patients was most likely attributable to a reduction of early mortality after initial rewarming.
Extracorporeal support is typically terminated immediately after rewarming in patients on standard ECC support because of bleeding, hemolysis, and inflammatory response. This is often possible only with high-dose catecholamine support and invasive ventilatory support. In contrast, by using ECMO, prolonged extracorporeal support is possible with minimal side effects until the cardiac and pulmonary functions of the patient have been stabilized. ${ }^{9,19}$ Furthermore, several studies have demonstrated that vasopressors are ineffective in hypothermia and therefore not recommended by international task forces. ${ }^{2,20} \mathrm{~A}$ high incidence of severe cardiopulmonary dysfunction is reported in most publications on extracorporeal rewarming of accidental hypothermia among both survivors and nonsurvivors. ${ }^{13,14}$

Vretenar and colleagues ${ }^{21}$ reported $60 \%$ survival among 68 hypothermic patients in a collective literature review; however, the high survival was explained to be the result of the publication bias of these types of studies and low rate of asphyxia-related cause of hypothermia. In addition, they reported that adult respiratory distress syndrome and severe pulmonary edema were the most frequent complications after successful weaning from ECC. However, in concordance with Vretenar and coworkers, Walpoth and colleagues ${ }^{13}$ and Locher and colleagues ${ }^{14}$ reported similar pulmonary complication rates among survivors and nonsurvivors of hypothermia. Thalmann and colleagues ${ }^{17}$ and Gilbert and colleagues ${ }^{22}$ reported on 2 hypothermic infants in cardiocirculatory arrest who were resuscitated by ECC, followed by ECMO support for several days because of weaning failure.

Severe pulmonary edema was frequently observed during the rewarming period of our patients. This phenomenon can be partly explained as ischemia-reperfusion injury but may be aggravated by severe diastolic dysfunction. Several studies on therapeutically induced hypothermia have reported severe stiffness of the left ventricle and acute volume overload during rewarming. ${ }^{23-25}$ If patients have to be weaned from ECC during refractory diastolic dysfunction with elevated left ventricular filling pressures, acute aggravation of reperfusion pulmonary edema occurs. Our study therefore underlines that prolonged ECMO support can prevent this complication if patients are weaned from ECMO after successful stabilization of cardiopulmonary function.

TABLE 4. Results of the multivariate logistic regression analysis to evaluate independent predictors for survival of accidental hypothermia with cardiocirculatory arrest $(n=59$ patients)

\begin{tabular}{llccc}
\hline & Wald & Relative risk & 95\% confidence interval & $\boldsymbol{P}$ value \\
\hline Asphyxia-related cause of hypothermia (avalanche, drowning) & 6.2 & 0.09 & $0.01-0.60$ & .013 \\
ECMO-assisted resuscitation & 4.2 & 6.61 & $1.2-49.3$ & .042 \\
Potassium at admission (per mmol/L) & 2.8 & 0.62 & $0.36-1.1$ & .09 \\
pH at admission & 0.018 & 0.89 & $0.02-83.8$ & .89
\end{tabular}

ECMO, Extracorporeal membrane oxygenation. 
18. Antretter H, Dapunt OE, Mueller LC. Survival of prolonged hypothermia. N Engl J Med. 1993;330:219.

19. Le Deist F, Menasche P, Kucharski C, Bel A, Piwnica A, Bloch G. Hypothermia during cardiopulmonary bypass delays but does not prevent neutrophil-endothelial cell adhesion. Circulation. 1995;92:354-8.

20. Schwarz B, Mair P, Wagner-Bacher H, et al. Neither vasopressin nor amiodarone improve CPR outcome in an animal model of hypothermic cardiac arrest. Acta Anaesth Scand. 2003;47:1114-8.

21. Vretenar DF, Urschel JD, Parrott JCW, Unruh HW. Cardiopulmonary bypass resuscitation for accidental hypothermia. Ann Thorac Surg. 1994;58:895-8

22. Gilbert M, Busund R, Skagseth A, Nilsen PA, Solbo JP. Resuscitation from accidental hypothermia of $13.7^{\circ} \mathrm{C}$ with circulatory arrest. Lancet. 2000;355:375-6

23. Velvis H, Hines MH, Klopfenstein HS, Berry DD, Vinten-Johansen J. Depression of cardiac function after deep hypothermic circulatory arrest in deeply anesthetized neonatal lambs. J Thorac Cardiovasc Surg. 1996;11:359-66.

24. Tveita T, Ytrehus K, Myhre ESP, Hevroy O. Left ventricular dysfunction following rewarming from experimental hypothermia. J Appl Physiol. 1998;85:2135-9.

25. Lauri T. Cardiovascular responses to an acute volume load in deep hypothermia. Eur Heart J. 1996;17:606-11.

26. Brugger H, Durrer B, Adler-Kastner L. On-site triage of avalanche victims with asystole by the emergency doctor. Resuscitation. 1996; 31:11-16.

27. Mair P, Kornberger E, Furtwängler W, Balogh D, Antretter H. Prognostic markers in patients with severe accidental hypothermia and cardiocirculatory arrest. Resuscitation. 1994;27:47-54

28. Schaller MD, Fischer AP, Perret CH. Hyperkalemia: a prognostic factor during acute severe hypothermia. JAMA. 1990;264: $1842-5$. 